\title{
Analysing the behaviour of robot teams through relational sequential pattern mining
}

\author{
Grazia Bombini ${ }^{1}$, Raquel Ros $^{2}$, Stefano Ferilli ${ }^{1}$, Ramon Lopez de Mantaras ${ }^{3}$ \\ ${ }^{1}$ University of Bari "Aldo Moro", Department of Computer Science, 70125 Bari, Italy \\ \{gbombini, ferilli\}@di.uniba.it \\ ${ }^{2}$ Department of Electrical and Electronic Engineering, Imperial College, UK \\ r.ros-espinoza@imperial.ac.uk \\ ${ }^{3}$ IIIA - Artificial Intelligence Research Institute, CSIC - Spanish Council for Scientific \\ Research, Campus UAB, 08193 Bellaterra, Spain \\ mantaras@iiia.csic.es
}

\begin{abstract}
This report outlines the use of a relational representation in a Multi-Agent domain to model the behaviour of the whole system. A desired property in this systems is the ability of the team members to work together to achieve a common goal in a cooperative manner. The aim is to define a systematic method to verify the effective collaboration among the members of a team and comparing the different multi-agent behaviours. Using external observations of a Multi-Agent System to analyse, model, recognize agent behaviour could be very useful to direct team actions.

In particular, this report focuses on the challenge of autonomous unsupervised sequential learning of the team's behaviour from observations. Our approach allows to learn a symbolic sequence (a relational representation) to translate raw multi-agent, multi-variate observations of a dynamic, complex environment, into a set of sequential behaviours that are characteristic of the team in question, represented by a set of sequences expressed in first-order logic atoms. We propose to use a relational learning algorithm to mine meaningful frequent patterns among the relational sequences to characterise team behaviours.

We compared the performance of two teams in the RoboCup four-legged league environment, that have a very different approach to the game. One uses a Case Based Reasoning approach, the other uses a pure reactive behaviour.
\end{abstract}

\section{Introduction}

Action selection in robotics is considerate a challenging task in different fields of Artificial Intelligence. An autonomous robot has to reason about the state of the environment and rationally act in order to complete a given task.

The complexity of each individual ability, and therefore the overall robots behaviour design, is related to the complexity of the environment where the robot carries out the task: the higher the complexity of the environment, the more 
challenging the robots behaviour design. Indeed, in real world unpredictable situations always occur, therefore is not possible to design completely controllable environments. Besides, creating highly controlled scenarios to decrease the difficulty of the task, makes it less realistic.

A robot needs multiple capabilities to perform a task, often divided into subtasks. For instance, to move a ball towards a goal point it should possess skills such as object detection, perception of the environment, building of an internal world model, making decisions when planning the task, navigation while avoiding obstacles, execution of planned actions, and recovering from failure. Thus, the reasoning engine must be capable of dealing with high uncertainty in the robots perception (incoming information of the world), and be robust in case of failure, since the outcomes of the actions performed are unpredictable. In this kind of environments, dynamic and unpredictable, the agent must be able to detect if the selected actions for a given state of the environment are still applicable when the state evolves. If they are, then the agent continues with the initial plan. Otherwise, it must either correct the selected actions or re-plan.

Moreover, in the case of a robot team, they have to jointly execute the selected actions, and coordinate among them to successfully perform the task. Based on the type and number of agents involved in the task the degree of difficulty of the task varies. The decision must be made in real time and in case of an autonomous robot as the ones in the Four-Legged League, with limited computational resources. Robot soccer is a particularly complex environment due to its dynamic nature resulting from the presence of multiple teammate and opponent robots. In particular, robots must agree on the decisions made, and who and what to do to complete the subtasks.

In general in multi-robot domains, and robot soccer in particular, collaboration is desired so that the group of robots work together to achieve a common goal. It is not only important to have the agents collaborate, but also to do it in a coordinated manner so that the task can be organized to obtain effective results. In this work we is address the problem of identification of collaborative behaviour in a Multi-Agent System (MAS) environment. The aim is to define a systematic method to verify the effective collaboration among the members of a team and compare the different multi-agent behaviours. Using external observations of a MAS to analyse, model, recognize agent behaviour could be very useful to direct team actions. In the analysis of such systems we have deal with the complexity of the world state representation and with the recognition of the agent activities. To characterise the state space, is necessary to represent temporal and spatial state changes occurred by agent actions.

Case-Based Reasoning (CBR) has been successfully applied to model the action selection of a team of robots in the robot soccer domain [1] (more precisely Four-Legged League). A case represents a multi-robot situation where the robots are distributed in terms of perception, reasoning, and action. The case-based retrieval and reuse phases are based on messages exchanged among the robots about their internal states, in terms of beliefs and intentions. Given a new situation, the most similar past case is retrieved and its solution is reused after some 
adaptation process to match the current situation. The case solution is modelled as a set of sequences of actions, which indicate what actions each robot should perform. This case representation ensures that the solution description in the cases indicates the actions the robots should perform; that the retrieval process allocates robots to actions; and finally, with the coordination mechanism, that the robots share their individual intentions to act. This approach allows for the representation of challenging rich multi-robot actions, such as passes in the robot soccer domain, which require well synchronized positioning and actions.

The $C B R$ approach is compared with the approach presented by the Carnegie Mellons CMDash06 team [2. In their approach they have an implicit coordination mechanism to avoid having two robots disputing the ball at the same time. The robot in possession of the ball notifies the rest of the team, and then the other robots move towards different directions to avoid collisions. The robots also have roles which force them to remain within certain regions of the field (for instance, defender, striker, etc.). The resulting behaviour of this approach is more individualistic and reactive in the sense that the robots always try to go after the ball as fast as possible and move alone towards the attacking goal. Although they try to avoid opponents (turning before kicking, or dribbling), they do not perform explicit passes between teammates to avoid them and in general they move with the ball individually. Passes only occur by chance and are not previously planned. Henceforward we will refer to this approach as the reactive approach.

This report is addresses the problem of learning and symbolically representing the sequences of actions performed by a teams of robots. The resulting relational representation of teams behaviours enable humans to understand and study the action concepts of the observed multi-agent systems and the underlying behavioural principles related to the complex changes of state space. A relational sequence could be used as a qualitative representation of a team behaviour. Low-level concepts of behaviour (events) are recognized and by these, high-level concepts (actions) are defined. Our proposal is to extract from raw multi-agent observations (log files) of a dynamic and complex environment, a set of relational sequences describing a team behaviour. The method is able to discover strategic events and through the temporal relations between them, learns interesting actions. The use of relational representations in this context offers many advantages. One of these is generalization across objects and positions. The set of the relational sequences has been used to mine the most frequent pattern. This reduced set represents the common sequences of actions performed by the team and represents the characteristic behaviour of a team. We also aim at selecting the most discriminative pattern to distinguish the reactive behaviour from the $C B R$ behaviour. 


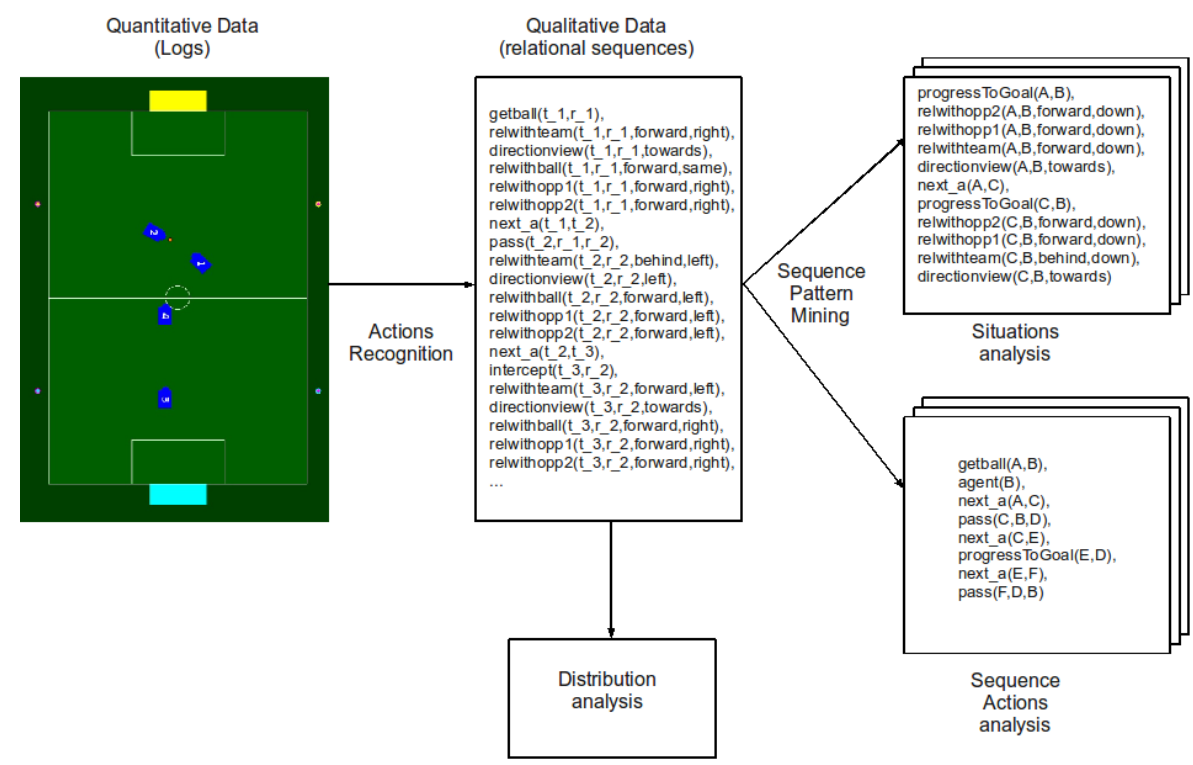

Fig. 1. Overview: generation of the relational sequences and the general phases of the analysis.

\section{Case Based Reasoning approach for action selection in the Robot Soccer Domain}

Using $C B R$ technique, the robots are able to perform explicit passes guided through cases. In $C B R$ new problems are solved by reusing and if is necessary adapting the solutions to similar problems that were solved in the past. A case represents a snapshot of the environment at a given time from a single robot point of view. This robot is called the reference robot, since the information in the case is based on its perception and internal state (its beliefs). The case definition is composed of three parts: the problem description, which corresponds to the state of the game; the knowledge description, which contains additional information used to retrieve the case; and finally, the solution description, which indicates the sequence of actions the robots should perform to solve the problem. More formally a case is defined as a 3-tuple:

$$
\text { case }=((R, B, G, T m, O p p), K, A)
$$

where:

1. $R$ : relative position wrt the ball and heading of the reference robot.

2. $B$ : balls global position.

3. $G$ : defending goal.

4. Tm: teammates relative positions wrt the ball. 
5. Opp: opponents relative positions wrt the ball.

6. $K$ : scope of the case defined as the regions of the field within which the ball and the opponents should be positioned in order to retrieve that case. With this representation, imprecision is easily handled since positions are relate to the regions instead of exact locations in the field.

7. A: sequence of actions (gameplays) each robot performs.

The first step in $C B R$ is the retrieval of past similar cases in order to reuse the solution of one of the retrieved cases. Similarity is evaluated along two important measures: the similarity between the problem and the case, and the cost of adapting the problem to the case. Thus, the features in the problem description are separated into two sets: controllable indices and non-controllable indices. The former refers to the reference robot and teammates positions (since they can move to more appropriate positions called adapted positions), while the latter refers to the balls and opponents position, and the defending goal, time and score (which is not possible directly modify). The idea of separating the features is that a case can be retrieved if we can modify part of the current problem description in order to adapt it to the description of the case.

The two main functions to evaluate cases are the following:

Similarity function: This measure indicates how similar the non-controllable features are between the problem and the case. Different functions for each domain of features are defined and then the overall similarity is computed using the harmonic mean of the individual similarities.

Cost function: This measure computes the cost of modifying the controllable features, i.e. the cost of adapting the problem to the case. It is defined as the sum of the distances between the positions of the robots in the problem and the adapted positions specified in the case after obtaining their correspondences. The adapted positions correspond to the global locations where the robots should be positioned in order to execute the solution of the case.

A subset of cases are manually created and stored in a file. When the system loads them, for each case the system automatically generates three more cases through spatial transformations taking into account the symmetries of the field. Since the considered domain is real time and because of computational limitations in the robots, it is essential to minimize the time invested during the retrieval process. To speed up the search, an indexed list is used to store the cases. Thus, given a new problem it is easy to access the subset of cases $\left(C B^{s}\right)$. Cases base are indexed using the value of the defending goal (yellow or cyan) and the number of opponents involved in each case.

After computing the similarities and costs between the problem and the cases in $C B^{s}$, a list of potential cases is obtained. To select the retrieved case, a compromise between the similarity degree between the problem and the case and the cost of adapting the problem to the case is considered. Moreover, since the domain is multi-robot (teams of robots), the cooperation between them is stimulated as much as possible. Therefore, the retrieval process orders the list of potential cases such that the similarity and number of players involved in the solution of the problem are maximized, while minizing the cost. The multi-robot 
system is composed of $n$ robots. All robots interact with the environment and among themselves, i.e. they perceive the world, they perform actions and they send messages to each other to coordinate and to exchange information about their internal states. Each robot has a copy of the same case base so they can gather the information needed during the case reuse.

Given a new state of the environment the first step is to select the robot responsible for the retrieval process and for the coordination of the robots during the case reuse. This robot is called the coordinator. The selection is based on the distance between the robots and the ball. The further a robot is from an object, the higher the imprecision about the objects information. Therefore, the coordinator corresponds to the one closer to the ball. Next, the coordinator retrieves a case according to the process described before and informs the rest of the robots which case to reuse.

At this point the case execution begins. Firstly, all robots that take part of the solution of the case move to their adapted positions. Once they reach them, they send a message to the coordinator in order to synchronize the beginning of the gameplay execution with the rest of the robots. Next, they all execute their actions until ending their sequences. Finally, they report the coordinator that they finished the execution and wait for the rest of the robots to end. When the coordinator receives all messages, it informs the robots so they all go back to the initial state of the process, i.e. selecting a new coordinator, retrieving a case and executing its solution. The robots may abort the execution of a case at any moment if any of the robots either detects that the retrieved case is not applicable anymore or an expected message does not arrive. In either case, the robot sends an aborting message to the rest of the robots so they all stop executing their actions and restart the process.

\section{Relational Sequential Pattern Mining}

In this section we present a method based on relational pattern mining, to extract meaningful frequent patterns able to define a behavioural team model. Here we briefly review the used representation language for the domain and induced knowledge. For a more comprehensive introduction to logic programming and Inductive Logic Programming (ILP) we refer the reader to [3].

A relational sequence is represented by a set of Datalog 4 atoms. A firstorder alphabet consist of a set of constants, a set of variables, a set of function symbols, and a non-empty set of predicate symbols. Each function symbol and each predicate symbol has an arity, representing the number of arguments the function/predicate has. Constants may be viewed as function symbols of arity 0 . An atom $p\left(t_{1}, \ldots, t_{n}\right)$ (or atomic formula) is a predicate symbol $p$ of arity $n$ applied to $n$ terms $t_{i}$ (i.e., a constant symbol, a variable symbols, or an $n$-ary function symbol $f$ applied to $n$ terms $\left.t_{1}, t_{2}, \ldots, t_{n}\right)$. A ground term or ground atom is one that does not contain any variables. A clause is a formula of the form $\forall X_{1} \forall X_{2} \ldots \forall X_{n}\left(L_{1} \vee L_{2} \vee \ldots \vee \bar{L}_{i} \vee \bar{L}_{i+1} \vee \ldots \vee \bar{L}_{m}\right)$ where each $L_{i}$ is a literal and $X_{1}, X_{2}, \ldots X_{n}$ are all the variables occurring in 
$L_{1} \vee L_{2} \vee \ldots \bar{L}_{i} \vee \ldots \bar{L}_{m}$. Most commonly the same clause is written as an implication $L_{1}, L_{2}, \ldots L_{i-1} \leftarrow L_{i}, L_{i+1}, \ldots L_{m}$, where $L_{1}, L_{2}, \ldots L_{i-1}$ is the head of the clause and $L_{i}, L_{i+1}, \ldots L_{m}$ is the body of the clause. Clauses, literals and terms are said to be ground whenever they do not contain variables. A Horn clause is a clause which contains at most one positive literal. A Datalog clause is a clause with no function symbols of non-zero arity; only variables and constants can be used as predicate arguments.

A substitution $\theta$ is defined as a set of bindings $\left\{X_{1} \leftarrow a_{1}, \ldots, X_{n} \leftarrow a_{n}\right\}$ where $X_{i}, 1 \leq i \leq n$ is a variable and $a_{i}, 1 \leq i \leq n$ is a term. A substitution $\theta$ is applicable to an expression $e$, obtaining the expression $e \theta$, by replacing all variables $X_{i}$ with their corresponding terms $a_{i}$. A conjunction $A$ is $\theta$-subsumed by a conjunction $B$, denoted by $A \preceq_{\theta} B$, if there exists a substitution $\theta$ such that $A \theta \subseteq B$. A clause $c_{1} \theta$-subsumes a clause $c_{2}$ if and only if there exists a substitution $\sigma$ such that $c_{1} \sigma \subseteq c_{2} . c_{1}$ is a generalization of $c_{2}$ (and $c_{2}$ a specialization of $c_{1}$ ) under $\theta$-subsumption. If $c_{1} \theta$-subsumes $c_{2}$ then $c_{1} \models c_{2}$.

A relational sequence is an ordered list of atoms. Given a sequence $\sigma=$ $\left(s_{1} s_{2} \ldots s_{m}\right)$, a sequence $\sigma^{\prime}=\left(s_{1}^{\prime} s_{2}^{\prime} \ldots s_{k}^{\prime}\right)$ is a subsequence (or pattern) of the sequence $\sigma$, indicated by $\sigma^{\prime} \sqsubseteq \sigma$, if

1. $1 \leq k \leq m$;

2. $\exists j, 1 \leq j \leq m-k$ and a substitution $\theta$ s.t. $\forall i, 1 \leq i \leq k: s_{i}^{\prime} \theta=s_{j+i}$.

A subsequence occur in a sequence if exists at least a mapping from elements of $\sigma$ ' into the element of $\sigma$ such that the previous condition are hold. In our case, that subsequence is a relational pattern.

The support of a sequence $\sigma$ in a set of sequences $\mathcal{S}$ corresponds to the number of sequences in $\mathcal{S}$ containing the sequence $\sigma$ : $\operatorname{support}(\sigma)=\left|\left\{\sigma^{\prime} \mid \sigma^{\prime} \in \mathcal{S} \wedge \sigma \sqsubseteq \sigma^{\prime}\right\}\right|$.

If we consider a sequence as an ordered succession of events, in our description language we distinguish two kinds of Datalog atoms: dimensional and nondimensional atoms. A dimensional atom explicitly refers to dimensional relations between events involved in the sequence. A non-dimensional atom denotes relations between objects (with arity greater than 1), or characterizes an object (with arity 1) involved in the sequence. In order to represent relational patterns, a dimensional operator must be introduced. We use next/2 to denote the direct successor operator. For instance, next $(\mathrm{x}, \mathrm{y})$ denotes that the event $\mathrm{y}$ is the direct successor of the event $\mathrm{x}$.

In order to mining the most frequent patterns, we use an Inductive Logic Programming (ILP) [5] algorithm, based on [6], for discovering relational patterns from sequences. It is based on a level-wise search method, known in data mining from the APRIORI algorithm [7]. It takes into account the sequences, tagged with the belonging class, and the $\alpha$ parameter denoting the minimum support of the patterns. It is essentially composed by two steps, one for generating pattern candidates and the other for evaluating their support. The level-wise algorithm makes a breadth-first search in the lattice of patterns ordered by a specialization relation. Starting from the most general patterns, at each level of the lattice the algorithm generates candidates by using the lattice structure and then evaluates the frequencies of the candidates. In this phase some patterns may be discarded 
due to the monotonicity of pattern frequency (if a pattern is not frequent then none of its specializations is frequent).

The generation of the patterns actually present in the sequences of the dataset is based on a top-down approach. The algorithm starts with the most general patterns. These initial patterns are all of length 1 and are generated by adding an atom to the empty pattern. Then, at each step it tries to specialize all the potential patterns, discarding those that do not occur in any sequence and storing the ones whose length is equal to the user specified input parameter maxsize. Furthermore, for each new refined pattern, semantically equivalent patterns are detected by using the $\theta$-subsumption relation and discarded. In the specialisation phase, the specialisation operator under $\theta$-subsumption is used. Basically, the operator adds atoms to the pattern. Finally, the algorithm may use a background knowledge $\mathcal{B}$ (a set of Datalog clauses) containing constraints on how to explore the lattice.

\section{Learning behavioural relational representation}

This section provides a description of the approach that we use to extract relational sequences from log files, which are able to describe and characterise the behaviour of a team of agents.

An example of a sequence of actions is presented in Figure 2. The four frames show a successful sequence of actions to overcome an opponent. The robot players are represented as blue boxes with a semicircular shape indicating body orientation. The attacking team is composed of robots 1 and 2, and the defending team of robots 3 and 4 (where robot 3 is the goalkeeper). The ball correspond to the orange circle.

The log used represents a stream of consecutive raw observations about each soccer player's position and the position of the ball at each moment of the trial. From this log streams it is possible to recognize basic actions (high-level concepts). Each team has sequences of basic actions used to form coordinated activities which attempt to achieve the team's goals. In our work, we identify the following basic actions of the players:

- getball $\left(T\right.$, Player $\left._{n}\right):$ at time $T$, Player $_{n}$ gains possession of the ball;

- $\operatorname{catch}\left(T\right.$, Player $\left._{n}\right):$ at time $T$, Player $_{n}$ gains possession of the ball previously belonging to an opponent;

- $\operatorname{pass}\left(T\right.$, Player $_{n}$, Player $\left._{m}\right):$ Player $_{n}$ kicks the ball and at time $T$ the Player $_{m}$ gains possession, and both players are from the same team;

- dribbling $\left(T\right.$, Player $\left._{n}\right)$ : at time $T$, Player $_{n}$ moves a significant distance avoiding an opponent; 

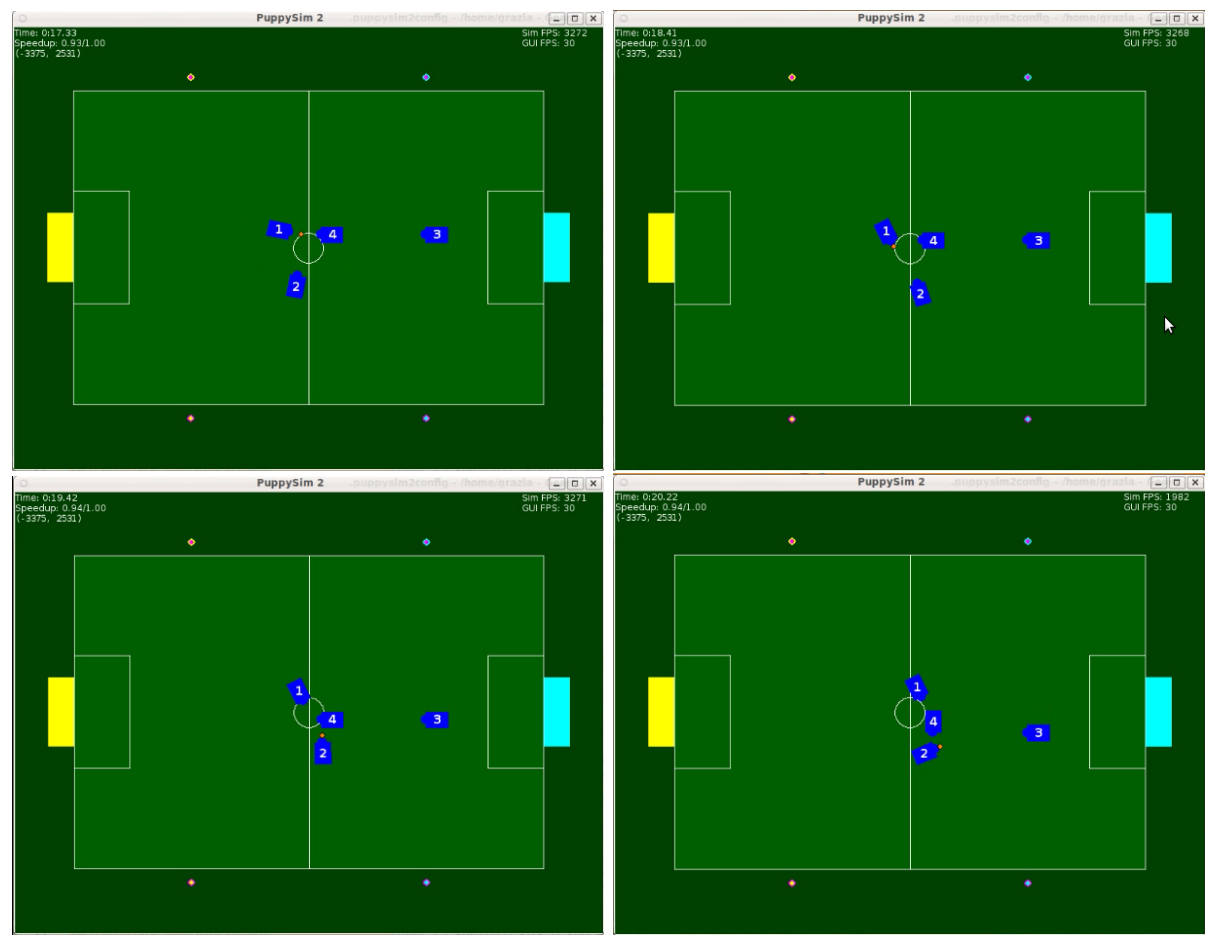

Fig. 2. An example of a sequence of actions

- $\operatorname{progressToGoal}\left(T\right.$, Player $\left._{n}\right)$ : at time $T$, Player $_{n}$ moves with the ball toward the the penalty box;

- aloneProgress ToGoal $\left(T\right.$, Player $\left._{n}\right)$ : at time $T$, Player $_{n}$ moves alone with the ball toward the penalty box, without teammate between it and the goal area;

- intercept $\left(T\right.$, Player $\left._{n}\right)$ : at time $T$, Player $_{n}$ loses the possession of the ball, and the new owner of the ball is from the opponent team;

The log stream is processed to infer the low-level events that occurred during a trial. An event takes place when the ball possession changes or the ball is out of bounds. Each next recognized event performed by a team contributes to form an action. To better describe the behaviour of an entire team, it is necessary to take into account the state of the word and the time in which the action is performed. Agents in dynamic environments have to deal with world representations that changes over time. A qualitative description of the world allows a concise and powerful representation of the relevant information. 
Each recognized event has some persistence over time and remains active until another event incompatible with it occurs. For example when a robot takes ball possession, an event indicating a new possession is generated. Subsequently, if an opponent manages to take possession of the ball, this generates a new event not compatible with the previous one and therefore it expires. An event that occurs in parallel with another event is called a contemporary event. For example, if an opponent tries to steal the ball from a robot that is in possession of it, the event of "trying to steal the ball" is contemporary to the event "ball possession". This contemporary event holds until one of the robots is able to take full possession of the ball, (i.e. moves away with the ball) or when the ball goes out of bounds.

A set of recognized events contributes to define an action. For example, when a player catches the ball, it could be due to a pass, an interception, or a dribbling, depending on the previous events still active. When an opponent tries to catch the ball, then a contemporary event occurs. In this case, if the ball did not belong to any robot for a while, then the system considers this situation to be a "getball". If the previous event was "catch of the ball" by the same robot and no opponent has attempted to take possession, then the system considers it a "aloneProgressToGoal", or a "progressToGoal" or a "getball" event, depending on the state of the world. In this situation, if a contemporary event is still held the system recognizes a "dribbling". If the previous event was the gain of the ball possession by an opponent, the the system considers this a "catch".

The current world state is represented by the positions of the players (teammates and opponents), and the ball. Instead of describing these through the actual coordinates or the identification of an area of the field, is more useful to describe the relation between these components of the state world. In this context, to characterise adequately specific scenes, we considered the viewpoint of the robot that performs the action, to determine how it interacts with others. This egocentric viewpoint has to rely on simple distinctions as, for instance, the position of another robot (a teammate or an opponent) that could be left or right of it, in front or back of it. More precise and objective descriptions would not reflect the generality and would not allow to abstract to positional information. Sequences represent a symbolic abstraction of the raw observation.

In particular, to describe the relation direction_view of the player with respect to the opponent's penalty box, we use front, left, right, backwards.

To describe the relation of the player with respect to the teammate, the ball and the opponents, we have used two arguments, one for the "horizontal" relation (forward or behind) and the other for the "vertical" relation (left or right). We use same when the player has the same position with respect to the teammate, the ball and the opponents.

- direction_view $\left(T\right.$, Player $_{n}$, position $)$;

- rel_with_ball(T, Player $_{n}$, horizontal, vertical $)$; 


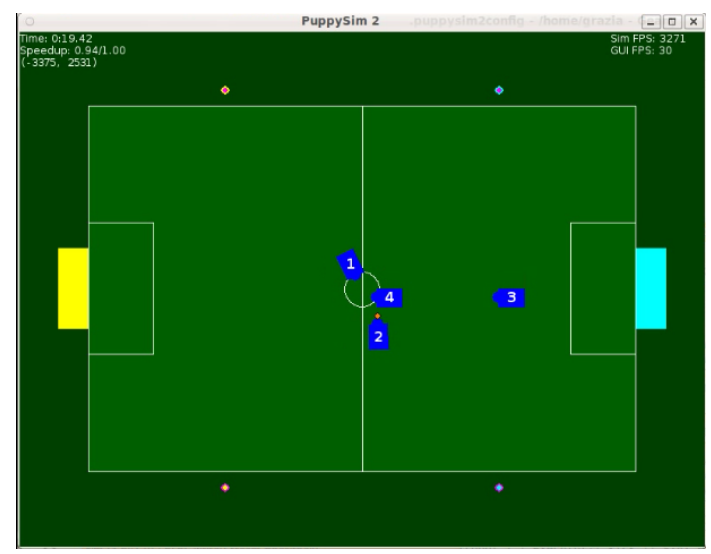

Fig. 3.

- rel_with_team $\left(T\right.$, Player $_{n}$, horizontal,vertical $)$;

- rel_with_opp1 $\left(T\right.$, Player $_{n}$, horizontal, vertical $)$;

- rel_with_opp2(T, Player $_{n}$, horizontal, vertical $)$;

For instance, the following predicate describes the environment depicted the Figure 3

pass $\left(\right.$ time $_{n}$, robot $_{1}$, robot $\left._{2}\right)$,

direction_view $\left(\right.$ time $_{n}$, robot $_{2}$, right $)$,

rel_with_team $\left(\right.$ time $_{n}$, robot $_{2}$, forward, left $)$,

rel_with_ball $\left(\right.$ time $_{n}$, robot $_{2}$, forward, same $)$,

rel_with_opp1 $\left(\right.$ time $_{n}$, robot $_{2}$, forward, right $)$,

rel_with_opp2 $\left(\right.$ time $_{n}$, robot $_{2}$, forward, right $)$

where opp1 represents robot 3 , and opp2 represents robot 4 .

The following predicates describe the result of the trial:

$-\operatorname{goal}(T)$ : at time $T$ the ball enters in the opponent's goal.

- to_goal $(T)$ : at time $T$ goes out of the field but passes near one of the goal posts.

- ball_out $(T)$ : at time $T$ the ball goes out of the field without being a goal or close to goal.

- $\operatorname{block}(T)$ : at time $T$ the goalie stops or kicks the ball.

- out_of_time $(T)$ : time out. 
This is an example of a sequence of actions and the environment description:

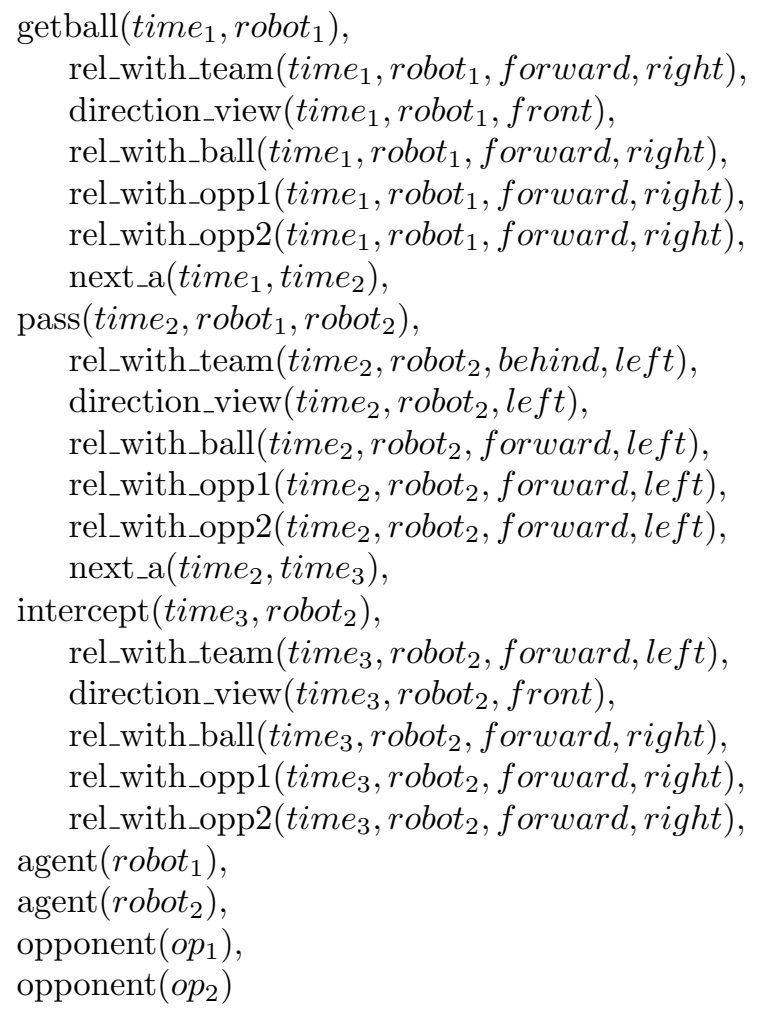

where $\operatorname{ext}_{a}\left(\right.$ time $_{n_{1}}$, time $\left._{n_{2}}\right)$ define the temporal relation between the actions.

\section{Experimental evaluation}

The aim of this experimentation is to measure and demonstrate the degree of collaboration of teams of soccer playing robots and, from a more general point of view, to characterise a Multi-Agent System behaviour. With the $C B R$ approach the performance of the robots should result in a cooperative behaviour where the team works together to achieve a common goal, a desired property in this kind of domain. Using a relational representation on the actions actually performed by a team, we intend to evaluate the collaborative behaviour of the team. Through the pattern mining method used, the most frequent set of behaviours is extracted. This set is able to characterise the behaviour of an entire team.

The $C B R$ approach allows the robots to apply a more deliberative strategy, where they can reason about the state of the game in a more global way, as well as to take into account the opponents. Thus, they try to avoid the opponents by passing the ball to teammates, which should increase the possession of the ball, and therefore, the team should have more chances to reach the attacking goal. 
As we will see, experiments revealed that these action sequences characterise the behaviour of the $C B R$ team. To be more precise, these action patterns are in the set of most significant patterns extracted from $C B R$ team sequences, whereas they are not among the most significant patterns extracted from the reactive (REA) team sequences.

We used an extended version of the PuppySim 2 simulator [1, created by the CMDash team [2]. This simulator represents the basic aspects of the RoboCup Standard Platform League, Four-Legged Soccer Competition [8]. Some additional features were implemented, such as managing team messages, robots walking while grabbing the ball, etc. The final version of the simulator is a simplified version of the real world. The robots perception is noiseless, i.e. the balls position and the location of all robots on the field is accurate. However the outcome of the actions the robots perform have a certain degree of randomness. The kicks are not perfect and the ball can end up in different locations within or around its ideal trajectory. In addition, when the robot tries to get the ball, it does not always succeed, simulating a "grabbing" failure (a very common situation with the real robots). The balls movement is modelled taking into account friction, starting with a high speed and gradually decreasing until stopping (if no one intercepts it before).

The case base used into the experimentation is composed of 136 cases. From this set, 34 cases are hand-coded, while the remaining ones are automatically generated using spatial transformations exploiting the symmetries of the soccer field.

The robots using the $C B R$ approach perform a default behaviour when no case is found. In these experiments, the default behaviour corresponds to the reactive approach. A simple behaviour for the opponents (defender, midfield defender and goalie) was implemented. Each robot has a home region and it cannot go beyond that region. If the ball is within its home region, then the robot moves towards the ball and clears it. Otherwise, the robot remains in the boundary of its home region, facing the ball to maintain it in its field of view. The experiments consist of two vs. two games.

\section{$5.1 \quad$ Setting}

Two sets of simulated experiments, one with the $C B R$ team and another one with the REA team, were performed taking into account two different scenarios. Besides, two possible configurations for the opponents are defined. The first is called DG configuration and considers a defender and a goalie. The second one, the $2 \mathrm{D}$ configuration, correspond to a midfield defender and a defender. The penalty area is reserved for the goalie, thus defenders are not allowed to enter into it.

In the 2D configuration each defender has its own home region with an overlapping area. The strategy of assigning regions to players is commonly used in robot soccer teams. In this way all the regions on the field are covered by at least one player and and the situation in which all robots chasing the ball is avoided. 


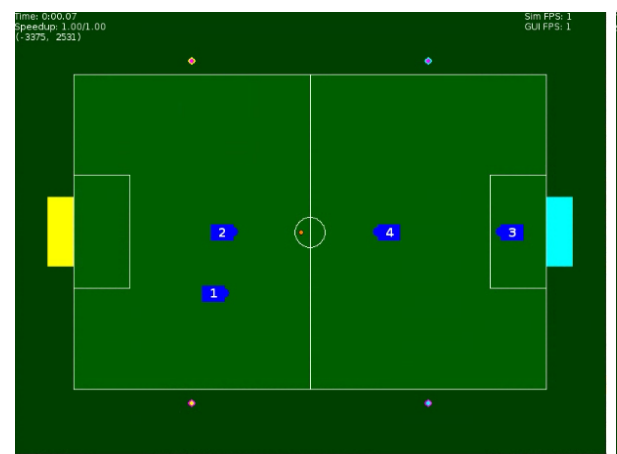

(a)

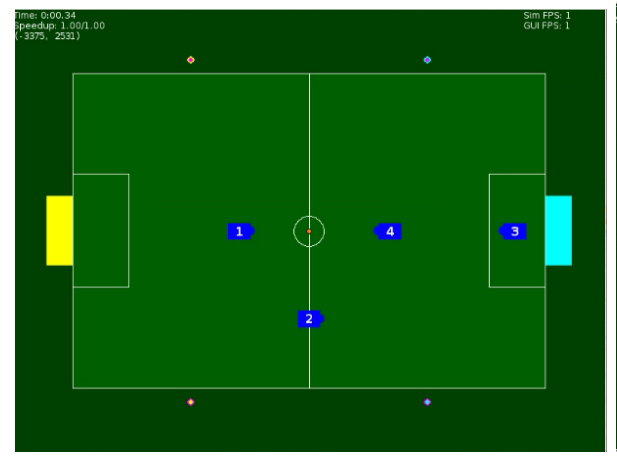

(c)

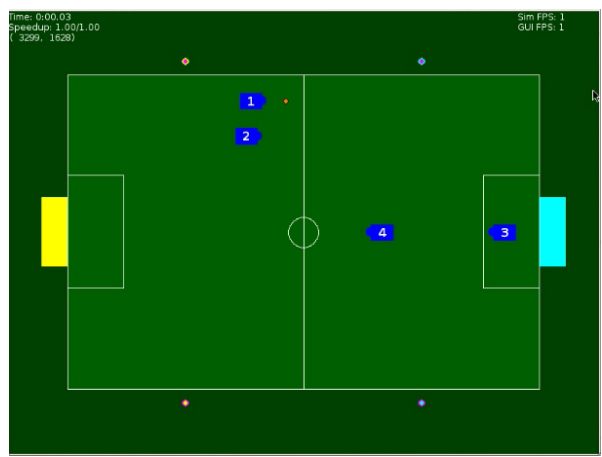

(b)

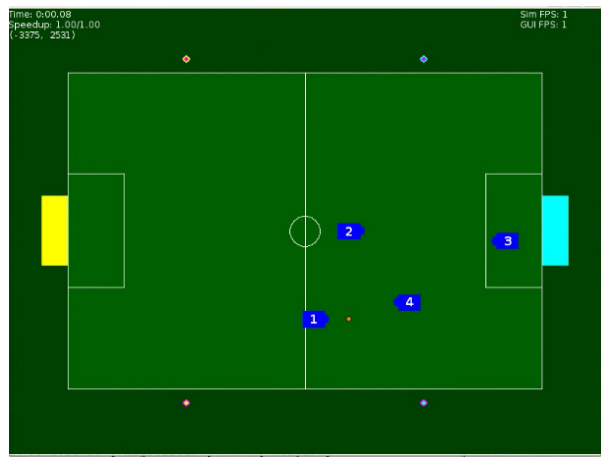

(d)

Fig. 4. Scenarios used during the experimentations with the DG configurations.

Four basic scenarios have been defined. These scenarios correspond to typical situations that usually occurs in football matches, where the attackers are coming from the back of the field towards the attacking goal, while the opponents are waiting at their positions. Each scenario is used with both configurations of opponents (DG or 2D). In scenario 1 (Figure 4 a) the ball and the attackers (robots 1 and 2) are positioned in the middle-back of the field, while in scenario 2 (Figure 4b), they are located in the left side of the field. The opponents (goalie, 3 , and defender, 4 , in these figures) remain within their home region. In the $2 \mathrm{D}$ configuration, the goalie is replaced with a defender.

In scenarios 3 and 4 (Figure 4 c and Figure $4 \mathrm{~d}$ ), the ball and attackers are located in the middle-front of the field. From a strategic point of view these scenarios are more interesting. The first decision (action) the attackers make (execute) is critical in their aim to reach the goal while avoiding the defender(s) whose main task is to intercept or to steal the ball.

These two sets of scenarios are general enough to represent the most important and qualitatively different situations the robots can encounter in a game. 
Initiating the trials on the left or right side of the field does not make much difference with respect to the actions the robots might perform in any of the two evaluated approaches, since they would perform the corresponding symmetric actions instead. We are interested in the defenders being active opponents complicating the attackers task. Thus, each scenario has been defined with the ball near the attacking goal, to allow defenders to attack and steal the ball.

The starting setting is fixed for any experimentation trial, but due imprecision of the actions the robots perform, the development of the trials varies from one to another. For this reason, during a trial very different and unpredictable situations may occur not known in advance.

\section{$5.2 \quad$ Results}

In order to evaluate our approach we analyse log files of soccer games. We have implemented a system that is able to identify and extract the interesting highlevel concepts and construct sequences of coordinated team behaviours using the recorded observations (logs) of this simulation games. The sequences have been defined on 7 atomic behaviours (catch, pass, dribble, etc.) and 5 environment descriptions (rel_with_ball, rel_with_team, etc.).

We performed 500 trials for each approach $(C B R$ and $R E A)$ and each scenario in the DG configuration, for a total of 4000 trials. From the raw observations of the log files we have obtained the dataset corresponding to this configurations. It is made up of 10261 sequences (6242 sequences $C B R$ approach and 4019 sequence for the reactive behaviour $R E A$ ).

Regarding the 2D configuration, we observed that the time required to end a trial was too long. This was due to the ability of the two defenders in preventing the attackers to reach the goal, not allowing them to reach the goal. For this reason a timeout to 60 second to end the trial was adopted. For the $2 \mathrm{D}$ configuration, we have performed 200 trials per scenario and per approach, for a total of 1600 trials. The dataset is made up of 4329 sequences(2392 sequences $C B R$ approach and 1937 sequence for the reactive behaviour $R E A$ ).

Data has analysed considering three dimensions: one that takes into account the distribution of actions recognized, a second one considering only the predicates that represent actions, and the last dimension, related to the environment in which actions were performed.

\subsection{Distribution of the actions}

In this section we analyse the distribution of the action recognized from the logs to better understand the behaviour of the entire team. Simply observing the composition of sequences is possible to make preliminary observations related to the degree of collaboration among robots. Modelling multi agent behaviour through relational sequences allows a good level of abstraction. Indeed, the distribution, in percentage, of actions recognized in all scenarios has the same characteristics (Figure 5) taking into account only the recognized actions. In particular, this 
Table 1. Recognized high-level concepts on DG configuration.

\begin{tabular}{|c|cc|cc|cc|cc|}
\hline & scenario A & scenario B & \multicolumn{2}{|l|}{ scenario C } & scenario D \\
& CBR REA & CBR REA & CBR REA & CBR REA \\
\hline N. sequences & 1595 & 977 & 1513 & 1170 & 1623 & 837 & 1511 & 1035 \\
\hline pass & 2285 & 1325 & 3135 & 557 & 2293 & 47 & 2023 & 190 \\
dribbling & 256 & 217 & 254 & 234 & 242 & 161 & 334 & 282 \\
catch & 5 & 3 & 24 & 3 & 10 & 0 & 7 & 0 \\
intercept & 1385 & 857 & 1306 & 1003 & 1434 & 771 & 1324 & 840 \\
aloneProgressToGoal & 216 & 35 & 291 & 44 & 192 & 34 & 177 & 34 \\
progressToGoal & 1261 & 585 & 974 & 1200 & 981 & 662 & 511 & 481 \\
getball & 2583 & 1246 & 2467 & 1423 & 2461 & 947 & 2272 & 1358 \\
\hline tot. Actions & 7991 & 4268 & 8451 & 4464 & 7613 & 2622 & 6648 & 3185 \\
\hline
\end{tabular}

Table 2. Recognized high-level concepts on 2D configuration.

\begin{tabular}{|c|cc|cc|cc|cc|}
\hline & \multicolumn{2}{|c|}{ scenario A } & \multicolumn{2}{|c|}{ scenario B } & \multicolumn{2}{|c|}{ scenario C } & scenario D \\
& CBR REA & CBR REA & CBR REA & CBR REA \\
\hline CB. sequences & 622 & 449 & 598 & 477 & 570 & 543 & 602 & 468 \\
\hline pass & 570 & 107 & 865 & 199 & 613 & 95 & 769 & 92 \\
dribbling & 77 & 83 & 85 & 73 & 84 & 65 & 63 & 99 \\
catch & 4 & 0 & 6 & 0 & 4 & 0 & 3 & 2 \\
intercept & 468 & 373 & 460 & 389 & 424 & 468 & 467 & 346 \\
alone & 34 & 9 & 24 & 17 & 34 & 13 & 40 & 22 \\
aloneProgressToGoal & 342 & 191 & 410 & 350 & 352 & 225 & 459 & 38 \\
progressToGoal & 883 & 508 & 907 & 605 & 801 & 682 & 896 & 524 \\
\hline tot. Actions & 2378 & 1271 & 2757 & 1633 & 2312 & 1548 & 2697 & 1123 \\
\hline
\end{tabular}

indicates that with this approach it is possible to define a model of the actual team behaviour.

As we can see in Table 1 and Table 2 the amount of sequences able to describe the behaviour of the team that uses the $C B R$ approach is significantly higher than the one that uses the reactive approach. Since the $C B R$ team plays using collaborative strategies, where the robots try to reach the goal area usually by passing the ball to a teammate, or moving to adapted positions to reuse the selected case, more sequences and therefore more actions are needed to describe such behaviour.

When the robot holding the ball tries to move towards the penalty area, having in front an opponent, it can act in a cooperative or individualistic way. That is, it can pass the ball to its teammate (in this case the recognized actions would be getball and pass) or could simply try to overpass the opponent, adopting an individualistic behaviour (if the robot succeeds in its aim, the action recognized will be a dribbling).

As can clearly seen in Figure 5 the number of the pass actions that appear in $C B R$ sequences is significantly higher than in $R E A$ sequences. The number of the dribbling actions that appear in the $R E A$ sequences is higher than in $C B R$ sequences. Actions like getball, progressToGoal and aloneProgressToGoal in- 


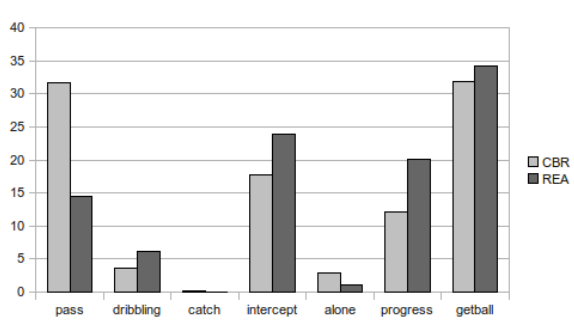

(a) DG configuration

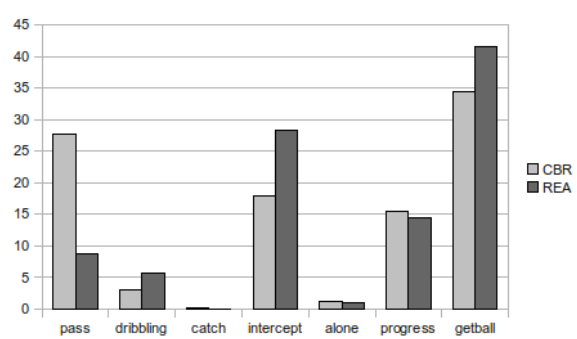

(b) $2 \mathrm{D}$ configuration

Fig. 5. Distribution in percentage of the actions on the entire dataset per configurations

dicate progress towards the goal area. The first two are more frequent in the $R E A$ team and this denotes a individualistic behaviour. Instead, actions such aloneProgressToGoal are more frequent in the case of $C B R$ sequences because, thanks to collaborative behaviour by means of passing, robots can overcome the defenders and progress alone towards the goal area, this is the only case in which a CBR robot moves alone since it has no teammate with whom collaborate. This is also true the case of $2 \mathrm{D}$ configuration in which we have two defenders, where the number of progressToGoal actions recognized in $C B R$ sequences is larger.

\subsection{Sequence Actions Analysis}

Here we consider the sequence analysis taking into account only the actions performed during the trials, without considering the predicates describing the state of the world. The goal of this experimentation was to find a subgroup of most meaningful patterns of actions able to characterise the behaviour of a team. We have used the whole dataset, all the sequences of the all scenarios per configuration. In particular, for the $D G$ configuration we have 10261 sequences, 6242 of which correspond to the $C B R$ and 4019 , to the $R E A$. Regarding the 2D configuration, the dataset is made up of 4329 sequences. In particular, we have 2392 sequences for $C B R$ and 1937 sequence for $R E A$.

To select the most meaningful patterns, i.e. a subset of frequent patterns that is able to characterise the essential behaviour of a team, we have used as measure the Fisher Score. The Fischer score 9 is popularly used in classification system to measure the discriminative power of a feature. In general, it is defined as

$$
F r=\frac{\sum_{i=1}^{c} n_{i}\left(\mu_{i}-\mu\right)^{2}}{\sum_{i=1}^{c} n_{i} \alpha_{i}^{2}}
$$

where $n_{i}$ is the number of data samples in class $i$, in our case the number of the sequences in class $i, \mu_{i}$ is the average feature value in class $i$, in our case is the average number of the occurrences of the pattern mined in class $i, \alpha_{i}$ is the 
Table 3. Some examples of most interesting patterns mined.

\begin{tabular}{|c|c|}
\hline pattern & Fisher score team \\
\hline getball $(\mathrm{A}, \mathrm{B})$, next_a $(\mathrm{A}, \mathrm{C}), \operatorname{pass}(\mathrm{C}, \mathrm{B}, \mathrm{D})$ & $0.23494427 \mathrm{cbr}$ \\
\hline $\operatorname{pass}(A, B, C)$, next_a $(A, D), \operatorname{getball}(D, C)$ & 0.07889064 \\
\hline progressToGoal $(A, B)$, next_a(A,C),intercept $(C, B)$ & 0.07138557 \\
\hline sToGoal $(\mathrm{A}, \mathrm{B})$, next_a $(\mathrm{A}, \mathrm{C}$ & 0.03037611 \\
\hline getball $(\mathrm{A}, \mathrm{B})$, next_a $(\mathrm{A}, \mathrm{C}), \operatorname{pass}(\mathrm{C}, \mathrm{B}, \mathrm{D})$, & \\
\hline $\begin{array}{l}\text { next_a }(\mathrm{C}, \mathrm{E}) \text {, il } \\
\text { getball }(\mathrm{A}, \mathrm{B}) \text {.n }\end{array}$ & 0.05987475 \\
\hline next_a $(C, D), \operatorname{pass}(D, B, E)$ & 0.05517990 \\
\hline getball(A,B),,next_a(A,C),pass(C,B,D), & \\
\hline next_a $(\mathrm{C}, \mathrm{E})$,progressToGoal $(\mathrm{E}, \mathrm{D})$ & 0.04290462 \\
\hline $\begin{array}{l}\text { progressToGoal }(A, B), \text { next_a }(A, C), \text { progressToGoal }(C, B) \text {, } \\
\text { next_a }(C, D) \text {,intercept }(D, B)\end{array}$ & 0.03860653 \\
\hline $\begin{array}{l}\text { progressToGoal(A,B),next_a(A,C),progressToGoal }(C, B) \text {, } \\
\text { next_a(C,D), progressToGoal(D,B) }\end{array}$ & 0.01199806 \\
\hline $\begin{array}{l}\text { getball(A,B),next_a }(A, C), p a s s(C, B, D), \text { next_a }(C, E), \\
\text { progressToGoal(E,D),next_a }(E, F), p a s s(F, D, B)\end{array}$ & 0.02503889 \\
\hline $\begin{array}{l}\text { getball(A,B),next_a }(A, C), \operatorname{pass}(\mathrm{C}, \mathrm{B}, \mathrm{D}), \text { next_a }(\mathrm{C}, \mathrm{E}) \text {, } \\
\text { getball(E,D),next_a }(\mathrm{E}, \mathrm{F}), \operatorname{pass}(\mathrm{F}, \mathrm{D}, \mathrm{B})\end{array}$ & 0.01994761 \\
\hline 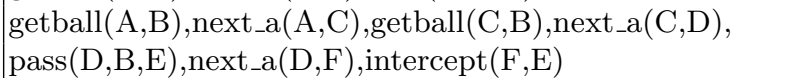 & 0.01829672 \\
\hline
\end{tabular}

standard deviation of the feature in class $i$, and $\mu$ is the average feature value in the whole dataset.

In 10 Cheng et al. demonstrate the frequency upper bound of discriminative measures such as information gain and Fisher score, showing a relationship between frequency and discriminative measures, and between the two discriminative measures. Since pattern of low support have a limited coverage of the dataset, these have a very limited discriminative power. But on the other hand, patterns of very high support have also a very limited discriminative power, since they are too common in the data. Therefore, in general it is appropriate to find patterns not too frequent with suitable support threshold. But this implies a greater effort during the pattern mining step.

Frequent patterns reflect strong association between objects, in this case represents common behaviours adopted by a team. The frequency is calculated over the whole dataset and over both sets of sequences ( $C B R$ and $R E A$ ). Among the different sequences for both teams, the most frequent patterns belong to the $C B R$ team. For this reason we have used the threshold $\sigma=0.10$, which is high enough to ensure adequate coverage of the dataset and sufficiently low to allow to discover frequent sequences also for the $R E A$ team.

Table 3 shows the most interesting patterns that have been extracted. As we can easily see, the presence of the predicate pass is enough to distinguish the $C B R$ team. Indeed, this type of action indicates collaborative behaviour, and is typical of sequences that can characterise the team $C B R$. 


\subsection{Situations Analysis}

The purpose of this analysis is to assess the state of the world when the action is performed. At the same state of the world, with the necessary abstractions related to the positions of the teammate, of the opponents and of the ball which are possible through a relational representation, follows a different action depending on the behaviour of the team. Here we consider the patterns mined taking into account predicates related to the description of the environment in which the agent acts. A pattern mined consists of sets of predicates such as rel_with_opp2(T, Player, horizontal, vertical), rel_with_opp1(T, Player, vertical, horizontal), rel_with_team(T, Player, horizontal, vertical) and so on, at the time of the action was performed. The mined set represents considerable information about the state of the world. Analysing this kind of situations is possible to abstract the difference between the behaviour of the two teams, the $C B R$ and the $R E A$.

For example in a situation in which the robot is in front of the penalty area and the teammate is in front, as well as the two opponents and the next action corresponds to a pass to the teammate is a typical sequence for the $C B R$ team with a significant Fisher Score:

Example 1. getball $(A, B)$, rel_with_opp $2(A, B$, forward, down $)$, rel_with_opp $1(A, B$, forward, down $)$, direction_view $(A, B$, front $)$, next_a $(A, C), \operatorname{pass}(C, B, D)$, rel_with_opp $2(C, D$, forward, down $)$, rel_with_opp $1(C, D$, forward, down $)$

In the same context, the characteristic sequence for the $R E A$ team that would be an interception. That is:

Example 2. getball $(A, B)$, rel_with_opp $2(A, B$, forward,up $)$, rel_with_opp $1(A, B$, forward, up $)$, next_a $(A, C)$, intercept $(C, B)$, rel_with_opp $2(C, B$, forward, up $)$, rel_with_opp $1(C, B$, forward, up $)$, direction_view $(C, B$, front $)$

This means that for the same situation, the $C B R$ team tries to overcome an opponent through a pass while, a team with a purely reactive behaviour tries to move towards the penalty area incurring more frequently in interception by the opponents. This indicates an individualistic behaviour. This analysis characterises the two different team behaviours and confirms the conclusions of previous analysis. 


\section{Conclusions}

In this report we have shown the potential of the use of a relational representation in a Multi-Agent domain to model the behaviour of the whole system. In this way it is possible to define a more high-level view of the behaviour on the multi-robot systems using a multi-agent activity logs.

In particular, this report is focused on the challenge of autonomous unsupervised learning of team behaviours based on observations. The aim was also to try to measure and demonstrate the degree of collaboration, analysing the joint behaviour of the teams. A desired property in a Multi-Agent systems is the ability of the team to work together to achieve a common goal in a cooperative manner. Our approach uses symbolic sequences (a relational representation) identification to translate raw multi-agent, multi-variate observations of a dynamic, complex environment, into a set of sequential actions that are characteristic of the team in question. The implemented method is able to discover strategic events and through the temporal relations between them learns the interesting actions. Raw multi-agent data logs were transformed into a set of sequential symbolic actions able to describe the team behaviour.

We compared the performance of two teams (REA and CBR) in the RoboCup four-legged league simulated environment, which have a very different behavioural approach. In general the results obtained in experiments confirm that the recognized action sequences characterise the behaviour of the teams. The set of relational sequences has been used to mine the most frequent patterns. This reduced set represents the common sequences of actions preformed by the teams.

\section{A Appendix A}

\section{Some examples of the most interesting patterns mined belonging to the $R E A$ team.}

- progressToGoal(A, B), rel_with_opp2(A, B, forward, down), rel_with_opp1(A, B, forward, up), rel_with_team(A, B, forward, up), direction_view(A, B, front), next_a(A, C), rel_with_opp1(C, B, forward, up), rel_with_team(C, B, forward, up), direction_view(C, B, front) 0.0406468

- progressToGoal(A, B), rel_with_opp2(A, B, forward, down), rel_with_opp1(A, B, forward, up), rel_with_team(A, B, forward, up), direction_view(A, B, front), next_a(A, C), pass(C, B, D), rel_with_opp2(C, D, forward, up), direction_view(C, D, left) 0.0404972

- progressToGoal(A, B), rel_with_opp2(A, B, forward, down), rel_with_team(A, B, forward, up), direction_view(A, B, front), next_a(A, C), pass (C, B, D), rel_with_opp2(C, D, forward, up), rel_with_opp1(C, D, forward, up), direction_view(C, D, left) 0.0369131 
- progressToGoal(A, B), rel_with_opp2(A, B, forward, down), rel_with_opp1(A, B, forward, up), rel_with_team(A, B, forward, up), direction_view(A, B, front), next_a(A, C), rel_with_team $(\mathrm{C}, \mathrm{B}$, forward, up), direction_view $(\mathrm{C}$, $\mathrm{B}$, front), progressToGoal(C, B) 0.0363782

- progressToGoal(A, B), rel_with_opp2(A, B, forward, down), rel_with_opp1(A, B, forward, up), rel_with_team(A, B, forward, up), direction_view(A, B, front), next_a(A, C), rel_with_opp1(C, B, forward, up), direction_view $(\mathrm{C}$, $\mathrm{B}$, front), progressToGoal(C, B) 0.0355654

- progressToGoal(A, B), rel_with_opp2(A, B, forward, down), rel_with_opp1(A, $\mathrm{B}$, forward, up), direction_view(A, B, front), next_a(A, C), pass (C, B, D), rel_with_opp2(C, D, forward, up), rel_with_opp1(C, D, forward, up), direction_view(C, D, left) 0.0348619

- progressToGoal(A, B), rel_with_opp2(A, B, forward, down), rel_with_opp1(A, B, forward, up), rel_with_team(A, B, forward, up), next_a(A, C), rel_with_opp1(C, B, forward, up), rel_with_team(C, B, forward, up), direction_view(C, B, front), progressToGoal(C, B) 0.0348601

- progressToGoal(A, B), rel_with_opp2(A, B, forward, down), rel_with_opp1(A, B, forward, up), rel_with_team(A, B, forward, up), direction_view(A, B, front), next_a(A, C), rel_with_opp1(C, B, forward, up), rel_with_team(C, B, forward, up), progressToGoal(C, B) 0.0348601

- progressToGoal(A, B), rel_with_opp2(A, B, forward, down), rel_with_opp1(A, $\mathrm{B}$, forward, up), rel_with_team(A, B, forward, up), direction_view(A, B, front), next_a(A, C), rel_with_opp1(C, B, forward, up), rel_with_team(C, B, forward, up), direction_view(C, B, front), progressToGoal(C, B) 0.0348601

- progressToGoal(A, B), rel_with_opp2(A, B, forward, down), rel_with_opp1(A, B, forward, up), rel_with_team(A, B, forward, up), next_a(A, C), pass(C, B, D), rel_with_opp2(C, D, forward, up), rel_with_opp1(C, D, forward, up), direction_view $(\mathrm{C}, \mathrm{D}$, left) 0.0334488

- progressToGoal(A, B), rel_with_opp2(A, B, forward, down), rel_with_opp1(A, $\mathrm{B}$, forward, up), rel_with_team(A, B, forward, up), direction_view(A, B, front), next_a(A, C), pass(C, B, D), rel_with_opp1(C, D, forward, up), direction_view(C, D, left) 0.0334488

- progressToGoal(A, B), rel_with_opp2(A, B, forward, down), rel_with_opp1(A, $\mathrm{B}$, forward, up), rel_with_team(A, B, forward, up), direction_view(A, B, front), next_a(A, C), pass(C, B, D), rel_with_opp2(C, D, forward, up), rel_with_opp1(C, D, forward, up) 0.0334488 
- progressToGoal(A, B), rel_with_opp2(A, B, forward, down), rel_with_opp1(A, B, forward, up), rel_with_team(A, B, forward, up), direction_view(A, B, front), next_a(A, C), pass(C, B, D), rel_with_opp2(C, D, forward, up), rel_with_opp1(C, D, forward, up), direction_view(C, D, left) 0.0334488

- progressToGoal(A, B), rel_with_opp2(A, B, forward, down), rel_with_opp1(A, $\mathrm{B}$, forward, up), direction_view(A, B, front), next_a(A, C), rel_with_opp1(C, B, forward, up), rel_with_team(C, B, forward, up), direction_view(C, B, front), progressToGoal(C, B) 0.0323264

- progressToGoal(A, B), rel_with_opp1(A, B, forward, up), rel_with_team(A, $\mathrm{B}$, forward, up), direction_view(A, B, front), next_a(A, C), rel_with_opp1(C, B, forward, up), rel_with_team(C, B, forward, up), direction_view(C, B, front), progressToGoal(C, B) 0.0307072

- progressToGoal(A, B), rel_with_opp2(A, B, forward, down), rel_with_team(A, B, forward, up), direction_view(A, B, front), next_a(A, C), rel_with_opp1(C, B, forward, up), rel_with_team(C, B, forward, up), direction_view(C, B, front), progressToGoal(C, B) 0.0303901

- progressToGoal(A, B), rel_with_opp1(A, B, forward, up), rel_with_team(A, $\mathrm{B}$, forward, up), direction_view(A, B, front), next_a(A, C), pass $(\mathrm{C}, \mathrm{B}, \mathrm{D})$, rel_with_opp2(C, D, forward, up), rel_with_opp1(C, D, forward, up), direction_view(C, D, left) 0.0300923

- progressToGoal(A, B), rel_with_opp1(A, B, forward, up), rel_with_team(A, B, forward, up), direction_view(A, B, front), next_a(A, C), rel_with_opp2(C, B, forward, down), rel_with_team(C, B, forward, up), direction_view(C, B, front), progressToGoal(C, B) 0.0250424

- getball(A, B), rel_with_opp2(A, B, forward, up), rel_with_opp1(A, B, forward, up), direction_view(A, B, front), next_a(A, C), rel_with_opp1(C, B, forward, up), direction_view(C, B, front), intercept(C, B) 0.0213223

- progressToGoal(A, B), rel_with_opp2(A, B, forward, down), rel_with_team(A, B, forward, up), direction_view(A, B, front), next_a(A, C), rel_with_opp2(C, B, forward, down), rel_with_team(C, B, forward, up), direction_view(C, B, front), progressToGoal(C, B) 0.0201827

- getball(A, B), rel_with_opp2(A, B, forward, up), direction_view(A, B, front), next_a(A, C), rel_with_opp2(C, B, forward, up), rel_with_opp1(C, B, forward, up), direction_view(C, B, front), intercept(C, B) 0.0197279

- getball(A, B), rel_with_opp2(A, B, forward, up), rel_with_opp1(A, B, forward, up), next_a(A, C), rel_with_opp2(C, B, forward, up), rel_with_opp1(C, 
B, forward, up), direction_view(C, B, front), intercept(C, B) 0.0197279

- getball(A, B), rel_with_opp2(A, B, forward, up), rel_with_opp1(A, B, forward, up), direction_view(A, B, front), next_a(A, C), rel_with_opp2(C, B, forward, up), rel_with_opp1(C, B, forward, up), direction_view(C, B, front), intercept $(\mathrm{C}, \mathrm{B}) 0.0197279$

- getball(A, B), rel_with_opp1(A, B, forward, up), direction_view(A, B, front), next_a(A, C), rel_with_opp2(C, B, forward, up), rel_with_opp1(C, B, forward, up), direction_view(C, B, front), intercept(C, B) 0.0194534

- getball(A, B), rel_with_opp2(A, B, forward, up), rel_with_opp1(A, B, forward, up), direction_view(A, B, front), next_a(A, C), rel_with_opp2(C, B, forward, up), rel_with_opp1(C, B, forward, up), intercept(C, B) 0.0194534

- getball(A, B), rel_with_opp2(A, B, forward, up), rel_with_opp1(A, B, forward, up), direction_view(A, B, front), next_a(A, C), rel_with_opp2(C, B, forward, up), direction_view(C, B, front), intercept(C, B) 0.0188371

- progressToGoal(A, B), rel_with_team(A, B, forward, up), direction_view(A, $\mathrm{B}$, front), next_a(A, C), pass(C, B, D), rel_with_opp2(C, D, forward, up), rel_with_opp1(C, D, forward, up), rel_with_team(C, D, forward, down), direction_view(C, D, left) 0.0188371

- getball(A, B), rel_with_opp2(A, B, forward, up), rel_with_opp1(A, B, forward, up), direction_view(A, B, front), next_a(A, C), rel_with_opp2(C, B, forward, up), rel_with_opp1(C, B, forward, up), direction_view(C, B, front) 0.0076135

Some examples of the most interesting patterns mined belonging to the $C B R$ team.

- getball(A, B), rel_with_team(A, B, behind, up), direction_view(A, B, backwards), next_a(A, C), pass(C, B, D), rel_with_opp1(C, D, forward, down), rel_with_team(C, D, forward, up), direction_view(C, D, right) 0.0281427

- getball(A, B), rel_with_team(A, B, behind, up), direction_view(A, B, backwards), next_a(A, C), pass(C, B, D), rel_with_opp2(C, D, forward, down), rel_with_opp1(C, D, forward, down), direction_view(C, D, right) 0.0257733

- getball(A, B), direction_view(A, B, backwards), next_a(A, C), pass(C, B, D), rel_with_opp2(C, D, forward, down), rel_with_opp1(C, D, forward, down), rel_with_team(C, D, forward, up), direction_view(C, D, right) 0.0245968

- getball(A, B), rel_with_team(A, B, behind, up), next_a(A, C), pass(C, B, D), rel_with_opp2(C, D, forward, down), rel_with_opp1(C, D, forward, down), 
rel_with_team(C, D, forward, up), direction_view(C, D, right) 0.0243266

- getball(A, B), rel_with_team(A, B, behind, up), direction_view(A, B, backwards), next_a(A, C), pass $(C, B, D)$, rel_with_opp2(C, D, forward, down), rel_with_team(C, D, forward, up), direction_view(C, D, right) 0.0243266

- getball(A, B), rel_with_team(A, B, behind, up), direction_view(A, B, backwards), next_a(A, C), pass (C, B, D), rel_with_opp2(C, D, forward, down), rel_with_opp1(C, D, forward, down), rel_with_team(C, D, forward, up) 0.0240568

- getball(A, B), rel_with_team(A, B, behind, up), direction_view(A, B, backwards), next_a(A, C), pass (C, B, D), rel_with_opp2(C, D, forward, down), rel_with_opp1(C, D, forward, down), rel_with_team(C, D, forward, up), direction_view(C, D, right) 0.0240568

- getball(A, B), rel_with_team(A, B, forward, down), direction_view(A, B, front $)$ next_a(A, C), pass $(C, B, D)$, rel_with_opp2(C, D, forward, down), rel_with_opp1(C, D, forward, down), direction_view(C, D, right) 0.0219150

- getball(A, B), rel_with_opp1(A, B, forward, down), rel_with_team(A, B, forward, down), direction_view(A, B, front), next_a(A, C), pass (C, B, D), rel_with_opp1(C, D, forward, down), direction_view(C, D, right) 0.0211173

- getball(A, B), rel_with_opp2(A, B, forward, down), rel_with_opp1(A, B, forward, down), direction_view(A, B, front), next_a(A, C), pass (C, B, D), rel_with_opp1(C, D, forward, down), direction_view(C, D, right) 0.0203171

- getball(A, B), rel_with_opp2(A, B, forward, down), rel_with_opp1(A, B, forward, down), rel_with_team(A, B, forward, down), next_a(A, C), pass (C, B, D), rel_with_opp2(C, D, forward, down), rel_with_opp1(C, D, forward, down) 0.0200586

- getball(A, B), rel_with_opp1(A, B, forward, down), direction_view(A, B, front), next_a(A, C), pass(C, B, D), rel_with_opp2(C, D, forward, down), rel_with_opp1(C, D, forward, down), direction_view(C, D, right) 0.0200527

- getball(A, B), rel_with_opp2(A, B, forward, down), rel_with_opp1(A, B, forward, down), direction_view(A, B, front), next_a(A, C), pass (C, B, D), rel_with_opp2(C, D, forward, down), rel_with_opp1(C, D, forward, down) 0.0195564

- getball(A, B), rel_with_opp2(A, B, forward, down), rel_with_opp1(A, B, forward, down), next_a(A, C), pass(C, B, D), rel_with_opp2(C, D, forward, down), rel_with_opp1(C, D, forward, down), direction_view(C, D, right) 
- getball(A, B), rel_with_opp1(A, B, behind, up), rel_with_team(A, B, behind, up), direction_view(A, B, backwards), next_a(A, C), pass (C, B, D), rel_with_opp1(C, D, forward, down), direction_view(C, D, right) 0.0185056

\section{References}

1. Ros, R., Arcos, J.L., Lopez de Mantaras, R., Veloso, M.: A case-based approach for coordinated action selection in robot soccer. Artif. Intell. 173(9-10) (2009) $1014-1039$

2. Veloso, M., Rybski, P.E., Chernova, S., Mcmillen, C., vonHundelshausen, J.F.F., Vail, D., Trevor, A., Hauert, S., Espinoza, R.R.: Cmdash05: Team report. Technical report, School of Computer Science, Carnegie Mellon University (2005)

3. Lavrac, N., Dzeroski, S.: Inductive Logic Programming: Techniques and Applications. Ellis Horwood, New York (1994)

4. Ullman, J.: Principles of Database and Knowledge-Base Systems. Volume I. Computer Science Press (1988)

5. Muggleton, S., De Raedt, L.: Inductive logic programming: Theory and methods. Journal of Logic Programming 19/20 (1994) 629-679

6. Esposito, F., Di Mauro, N., Basile, T., Ferilli, S.: Multi-dimensional relational sequence mining. Fundamenta Informaticae 89(1) (2008) 23-43

7. Agrawal, R., Mannila, H., Srikant, R., Toivonen, H., Verkamo, A.: Fast discovery of association rules. In: Advances in Knowledge Discovery and Data Mining. (1996)

8. Committee, R.T.: Robocup four-legged league rule book. (2008)

9. Duda, R.O., Hart, P.E., Stork, D.G.: Pattern Classification (2nd Edition). WileyInterscience (November 2000)

10. Cheng, H., Yan, X., Han, J., wei Hsu, C.: Discriminative frequent pattern analysis for effective classification. In: In ICDE. (2007) 716-725 